\title{
Astronomy, Technology, and the Scientific Method: Reflections on the Astronomy Research Seminar at Stanford Online High School, Spring 2018
}

\author{
Kalee Tock ${ }^{1 *}$
}

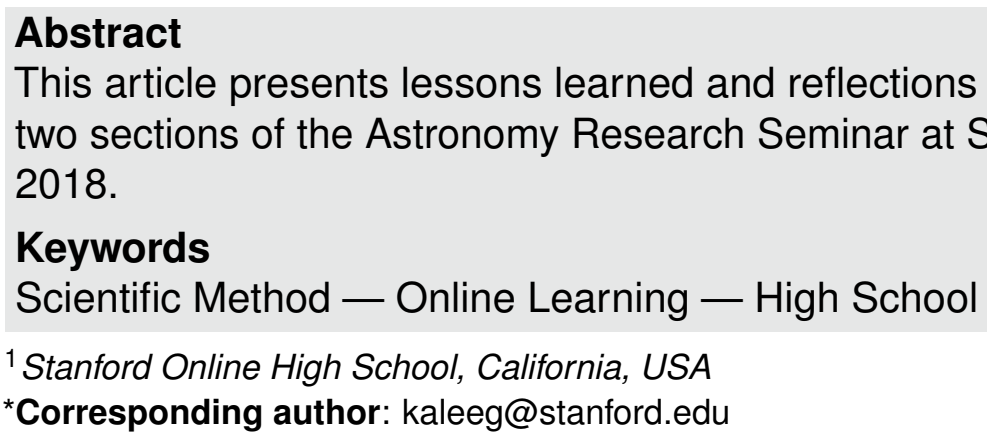

\section{Introduction}

Stanford Online High School (SOHS) does not fit the picture that most people have of online education. Unlike large, anonymous, Massive Open Online Courses (MOOC's), SOHS classes are small and synchronous. We meet online twice weekly in seminar groups of no more than 16 students. Students are marked tardy if they are late, and are graded on their participation in the live discussion. We have an active community of intellectually passionate students, many of whom are engaged in significant outside pursuits. In this context, a "significant outside pursuit" has a large associated time commitment that would make participation in a brick-and-mortar school difficult. SOHS is home to dancers, actors, equestrians, musicians, and athletes of every description. Although classes meet online, there are several in-person meetups and activities that take place throughout the year. Figure 1 shows some students getting together to solve puzzles on an astronomy-themed spring break trip that took place in March of 2018.
It is in this context that I initiated the Astronomy Research Seminar in spring of this year. I preceded the official course with two extracurricular pilot projects on double stars and eclipsing binaries, as proof of concept that extended research projects process were possible in this environment with these students.

\section{Course Structure}

Like all SOHS science courses, the Astronomy Research Seminar met twice weekly for 70 minutes per meeting. However, the two sections that I taught were very different. One of the sections was taught through the Malone School Open Network (MSON), a consortium of independent schools throughout the United States, of which SOHS is the sole wholly-online school member. The MSON section consisted of only four students, from three different schools. This group decided to take on a double-star research project all together, as a single section, and they completed a compare-contrast study of four different star systems. Because I was in attendance for the entirety of all of their project group meetings, I took an active role in leading and organizing the project. This group finished and submitted their 


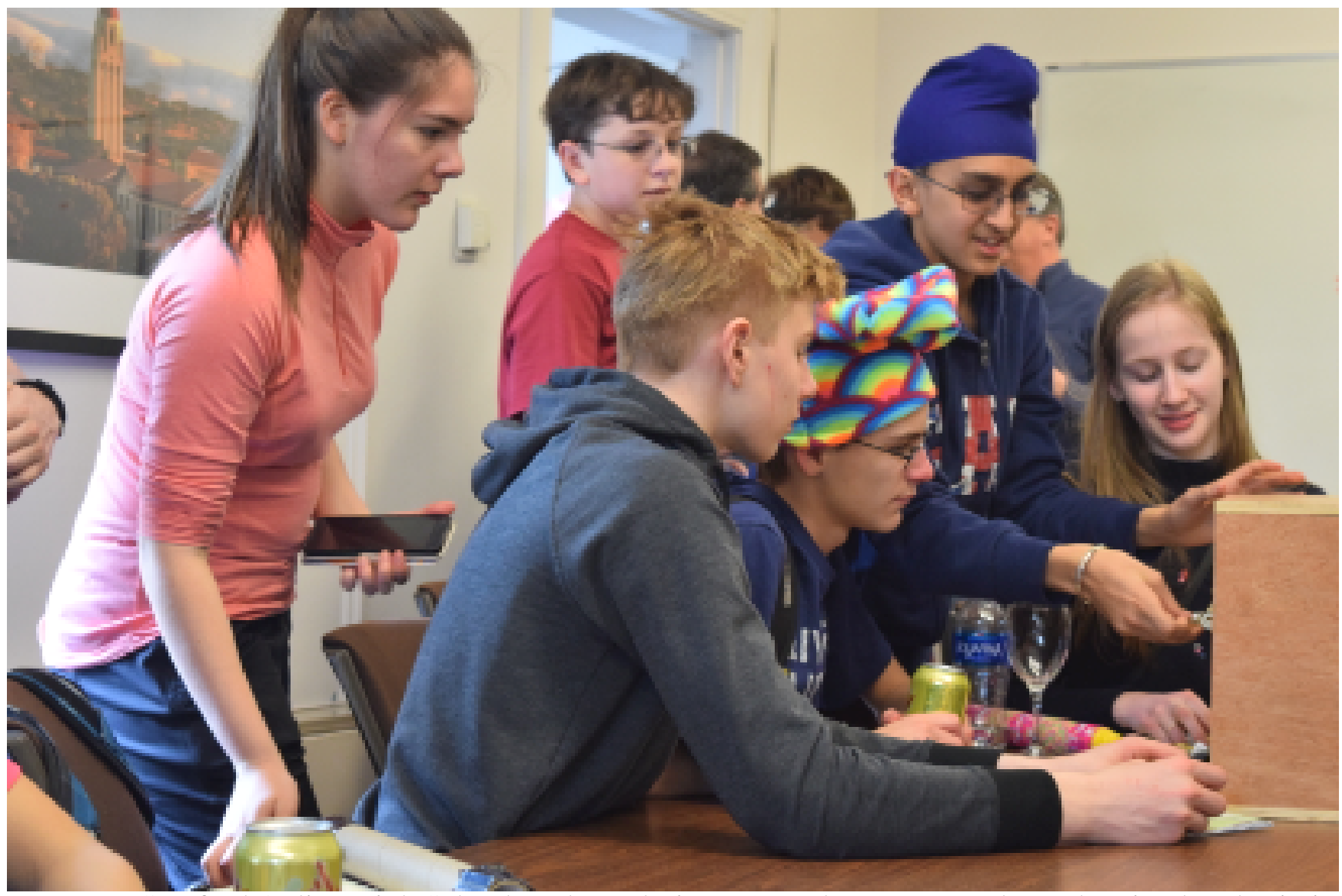

Figure 1. Stanford Online High School students during a 1 week astronomy-themed spring break trip in March 2018, unlocking a box at the end of a solar system treasure hunt

paper early in the spring, and we spent the remaining weeks of the semester doing small side projects, not for publication.

The second section, from SOHS, had 13 students, split into 5 teams. Three of the teams did double-star projects and two did eclipsing binary projects. Almost all of the section meeting time took place in project breakout rooms, with me popping in on as many breakouts as I could during the 70-minute class meeting. As a consequence, roughly $4 / 5$ of each project's group meeting time was completely self-directed by the students. Only one of the groups from this second section finished early, and at the time of this writing, some of the groups are still completing their projects.

\subsection{The Efficiency of Self-Directed Projects}

There are several reasons for the decreased efficiency of the self-directed SOHS project groups. First, students do not have enough experience with astronomy research to determine the best way forward in a scientific project. "What should we do next?" was the most common question I heard when I visited their breakouts. Although there is rarely one right answer to this question, the students didn't know any of the possible answers, and often spent significant time discussing ideas that were unworkable for one reason or another, or sending each other off on impossible quests for information or measurements. Additionally, the lack of a clear project lead within each group, and the reluctance of students to hold their peers accountable for contributions to the project, made for a slower pace. Because of the many, known difficulties with group work in educational environments, I made each student's weekly grade dependent on a writeup of their individual project contributions, which was submitted directly to me. (This is something that I'd like to change for next year, because it proved difficult to assess. At 


\section{Astronomy, Technology, and the Scientific Method: Reflections on the Astronomy Research Seminar at Stanford Online High School, Spring 2018 - 3/7}

the time of this writing, I remain unsure how best to adjust the metric.) In any case, although the SOHS groups made slower progress and spent considerably more time flailing than the MSON group, their learning experience was a more authentic representation of actual science. At least, that's what I'm telling myself.

\section{The Scientific Process in Research vs in In- troductory Classes}

Over the course of the semester, I found myself reflecting often on the scientific process and the ways in which actual research differs from the picture we paint for students in introductory science classes. A quote from Joe Madeiro, JPL engineer, encapsulates some of these disparities. Dr. Madeiro spoke to our group during the astronomy spring break trip referenced above.

"Anytime you do a new astronomical survey you get new data, and you find new things: things you can't anticipate. . . . When you build a new telescope, you have to have a reason to build it. You can't just build it because it 'feels right'. But you should know that of the reasons you build something, maybe half of them won't be interesting anymore by the time it gets built. However, 10 times as many more things will come along that you could never have anticipated. A survey was done with the Hubble Space Telescope, which was proposed in the 60's and 70's, finally launched in the 80's or 90's, repaired, etc. When Hubble was built there were 10 main goals that they wanted to accomplish. Later they asked themselves, "How have we done with the original goals, and what are the most impactful things that have come out of this?" And three or four of the original goals turned out to be, as expected, some of the main science that has ever come out of Hubble. But, 7 of the top 10 goals were not in the 'most impactful' list." (Madiero, in person talk in Pasadena, March 2018)

My takeaway from this is that in real science, you have to be open to pursuing other paths than the one upon which you originally set out. The experiments that we do in most of our science classes run counter to this, because we grade students on their answers to a specific question. If the lab asks you to cushion the fall of an egg, you will not get credit for investigating the optical properties of the saran wrap you used, even though this might ultimately be more interesting or impactful than the experiment that was assigned. At best, we'll give you a nod and a "hey, that's cool" but as instructors, what we will think hard about and assess and give meaningful feedback on is the efficacy of your egg cushion.

This is how it has to be in an introductory setting because 1) there are not enough hours in the day for instructors to guide 60 students pursuing 60 different experiments, no matter how awesome these might be, 2) students (mostly) don't yet have the knowledge and experience to be able to predict whether the topics they want to investigate have the potential to bear real scientific fruit, and 3) instructors would get complaints about "lack of clarity" for including open-ended goals their lab protocols (ask me how I know). So, it would be impossible to completely replace traditional labs with indiscriminate experimentation for purposes of a normal class, though we can take little steps like encouraging procedural creativity within constraints and encouraging students to keep track of their tangential ideas in sidebars (e.g. requiring a "Notes for Future Research" section).

While the place of student innovation in classroom lab experiments is limited at best, this sort of inventiveness plays a huge role in the scientific enterprise. Real, impactful science depends on scientists' being ready to intentionally study different, more interesting questions than the ones they set out to ask. It would be difficult to train students to "keep their eyes open" the way they would need to do as scientists. But, we do them a disservice in pretending that the cycle of hypothesis - data - conclusion they follow in traditional lab experiments mimics the way science is actually done. Concentrating on cushioning the egg instead of sidetracking into the nuances of how saran wrap interacts with light is what students must do in order to earn an A in our classes, but in "real life", the scientist should find a way to pursue the plastic optics experiment in addition or even instead.

Students who succeed in the artificial environ- 


\section{Astronomy, Technology, and the Scientific Method: Reflections on the Astronomy Research Seminar at Stanford Online High School, Spring 2018 - 4/7}

ments we create with classroom lab experiments are often not the ones who succeed in real world problem-solving environments, where the questions are less well-defined and the answers are murkier. Indeed, studies have found an inverse relationship between "students' reported GPA and their orientation toward creative or innovative work", which is why Google and other companies no longer ask for transcripts when hiring employees (Gray 2016). As an instructor, I sometimes encounter indignation when I ask students to solve problems creatively. For example, if I set the task of figuring out a way to measure the volume of a system or deciding how to present numerical data graphically, I might be told that my protocol was confusing or that it was not clear "what we were supposed to do." Students who are apprehensive about taking intellectual risks in these sorts of limited-scope situations will be even less willing to be creative about which questions to ask in the first place.

In summer of 2018, I took an astrophysics and fusion teacher workshop at Lawrence Livermore National Laboratories, and on one of the days we toured the Jupiter laser facility. The physicist who took us around explained that research groups come from all over the world to use the lasers there. He said that often the equipment does not function as expected or intended, and groups have to find a way to make productive use of the valuable beam time that they secured for their project. In some cases, they end up doing completely different science, simply because of which lasers are functioning at the time of their run. Groups that can roll with the punches, troubleshooting and fixing things and finding alternatives to their original goals, are (in his words) "the ones we want", because those researchers eventually become the most successful scientists.

This seems to be a common theme, and it calls to mind the many twists and turns of my Astronomy Research Seminar projects this semester. For the eclipsing binary projects, we sidetracked into an exploration of various photometric methods, invented ways of classifying images before analysis, and investigated multiple period-finding algorithms instead of determining the temperature of the system as we had originally set out to do. For the double star projects, we became deeply entangled in coming up with a mathematical technique to infer the proper motion of a secondary star from that of the primary plus the secondary's relative motion. We also sidetracked into understanding how to use an "improvement parameter" (invented by the course TA) to assess trends in the residuals of an orbital solution (Crigler et al. 2019).

Particle physicist Don Lincoln has a video in which he says: "Without transistors, the computer revolution would have never happened. Without particle accelerators, there would be no radiation treatment for cancer. Without the development of large accelerators with superconducting magnets, it would have been a long time before medical MRI magnets would have been available. Even more recently, particle physicists can point to the World Wide Web, which was originally designed to facilitate communication between researchers . . . ". Lincoln cites these examples to make the point that particle physics is worth funding. And it is certainly true that these advances would not have happened without particle physics. But more importantly, they would not have happened if the particle physicists had been constraining themselves to answer only the questions they were asking about the subatomic particles they were studying.

I think it is important to emphasize to students that in our classroom lab experiments, we are teaching them scientific techniques, but we are not "doing science". We are giving them practice with having a guiding question, just as the designers of the HST had clear questions that they hoped to use the telescope to answer when it was launched. But, we are not giving them practice with the fundamental and ultimately more important skills of being creative and being able to decide when to purposefully switch gears, asking different questions and making connections that are tangentially (or not at all) related to the original phenomenon under study. For purposes of the introductory classroom, we must insist that students stay focused on the assignment and answer the original question because we are teaching them to use specific tools. But we should be clear with students that if a chemistry lab 


\section{Astronomy, Technology, and the Scientific Method: Reflections on the Astronomy Research Seminar at Stanford Online High School, Spring 2018 - 5/7}

involves a titration, the learning goal is "how to do a titration," not "how to do science".

Unlike other classes I've taught, the learning goal for the Astronomy Research Seminar is "how to do science". The students who have accumulated the most tools from previous math / science / CS classes often have an advantage; they can make the most connections because they have the biggest reservoir of prior experience to make connections between. But more important is the ability to be self-directed, and this is not a skill that is honed by make-a-measurement, learn-a-skill labs. As a consequence, students become uneasy and frustrated by tasks that are not clearly-defined, setbacks that are unforeseen, and circumstances make the original goals difficult or impossible to achieve. In truth, scientists become frustrated by such things also. The difference is that they do not view the difficulties as inappropriate. The outlook changes everything.

Every single one of my Astronomy Research Seminar projects this semester veered off-course from the direction taken at the outset. The distractions took various forms, but they all had them. We certainly did not made the best possible choice of sidetracks in all or even most cases. Were we to start over, I would advise doing things differently in pretty much every project. That is the nature of the beast. I was fortunate that the 13 students who signed up for my first semester of teaching the Astronomy Research Seminar were (for the most part) students who could handle the uncertainty, though many of them did tell me that it represented a sharp departure from science classes they had taken in the past.

Just to be clear, I'm not suggesting that we completely replace traditional science labs with self-directed experimentation of the Astronomy Research Seminar variety. The skills that are developed by means of traditional labs are important, and students need as many tools as we can give them. For example, they'll never think to use a titration as a means of probing a system they are studying unless at some point they've had the makea-measurement, learn-a-skill experience of doing one. So, we cannot and should not do away with these sorts of activities in our introductory science classes. We just need to be more explicit about what they are, and incorporate some more open-ended "real science" components into our introductory curricula where possible.

\subsection{The "Scientific Method" as Commonly Taught and Practiced in Introductory Classes}

In addition to differentiating classroom experiments from actual science, I believe that we should teach the scientific method differently. The scientific method is usually the first unit in a science class, and students snooze through it because they have been hearing about it since elementary school. It prefaces the make-a-measurement, learn-a-skill labs that they will be doing for most of the year and brushes aside the most important and most impactful part of those experiments: the slight adjustments that students end up making to the protocol to "get things working." As a consequence, students completely omit these from their lab writeups.

For example, in a microbial fuel cell lab, yeast are suspended in a mixture of lime jello in order to test the voltage across the mixture. A student forgot to mix in the yeast, and the jello had set in the fridge before he remembered. He re-melted the jello, monitored the temperature, mixed in the yeast, and let it set a second time in the fridge. None of this was evident from his writeup. To all appearances, he had mixed in the yeast before the jello first set, as per the lab protocol. His voltage results were slightly different from those of other students in the class, but for lack of documentation, a reader would have no reason to suspect that any difference in his procedure might have affected this.

In a DNA extraction lab, a student initially did not have the correct concentration of alcohol, and tried the procedure with the lower concentration that she had in her cabinet. She was unable to see the extracted DNA, so she bought the higher concentration and tried that. At first, that didn't work either. Eventually, she figured out that the alcohol wasn't cold enough to crystallize the DNA, so she extended its time in the freezer and tried a third time. Ultimately, this was successful: she was able to see and photograph the strands of crystallized DNA on a toothpick. But once again, none of this appeared 


\section{Astronomy, Technology, and the Scientific Method: Reflections on the Astronomy Research Seminar at Stanford Online High School, Spring 2018 - 6/7}

in her writeup. Reading her report, a reader would think she had done the experiment only once, using the recommended concentration of alcohol, and would not know how the time the solution spent in the freezer had been adjusted.

These examples represent work done by good students. They knew the "scientific method" and would ace any question about it that might come up on a test. I only know about the differences between their writeups and their actual procedures because they asked me to read drafts of their reports in office hours; in the course of doing so, I asked them questions about what they had done. They were surprised that I would be at all interested in these sorts of details and even more surprised when I insisted on including them in their writeups. And these are only two of many, many more examples of similar omission of "trivialities" that do not conform to the idealized picture of how science is done, which students bring to our classrooms and which we unknowingly reinforce with that first "scientific method" unit.

Students often come to my office hours to ask me to look over their lab writeups before they turn them in. I'll read it out loud to them, asking questions along the way. Every single time, asking questions reveals that their story is incomplete. There was some adjustment that they made due to their particular circumstances that they didn't think was "important" enough to document. Or, their first try "didn't work", and rather than analyzing it or even documenting it at all, they threw it away, did the experiment a second (or third) time, obtained the expected results, and documented that instead. The instances I know about from office hours are only the tip of a much larger iceberg. In passing on the class Skype group, in talking to parents at parentteacher conferences, and on the last day of class when we share memorable moments from experiments done over the course of the year, I hear many more such stories.

With this mindset, Alexander Fleming would have thrown away his moldy petri dishes instead of looking closer, thinking harder, and ultimately discovering antibiotics. Darwin would have stayed focused on the plants he was studying rather than realizing that the mockingbirds on the islands he visited constituted an important clue to a different puzzle. Penzias and Wilson would have ignored the faint noise in their radio receiver rather than using it to track down the cosmic microwave background. And had they stayed true to "the scientific method," my student project groups would have foregone some of the most interesting science that they ended up doing in the Astronomy Research Seminar this past spring.

\section{Proposal for More Effective Instruction in the Scientific Method}

With our curricular focus on the experimental outcomes we anticipate, instructors unintentionally strengthen this tendency to dismiss unexpected results and tangential interesting questions. To counter this, I propose putting the "how science works" parts of the curriculum not as the first unit of a course, but in the middle, or even last, ideally after students have done some sort of self-directed project in which they experienced significant roadblocks or changes in direction. Many students come to our classes with a conception of the scientific process that we simply cannot dispel with citations of history or exhortations to include their missteps in their lab documentation. I've tried all kinds of gimmicks, from insisting that they troubleshoot a non-working circuit (even if their LED did light up the first time they put it together), to refusing to let them discount aberrant measurements without analysis, to assigning a point value to the documentation of an unexpected outcome. They'll do it if it is part of their grade. But, as soon as they are not earning points for it, initial missteps, tangential observations, and unexpected results suddenly and magically stop happening, because these do not fit the picture we have painted of how science is meant to be done. It is only after students have experienced a significant roadblock themselves that they stand to gain a deeper understanding of the importance of such hindrances.

As McDermott et al. write in Preparing Teachers to Teach Physical Science By Inquiry, "The scientific process can only be taught by direct experience." (McDermott et al. 2000) Experience enables 
one to arrange ideas more hierarchically and into fewer categories, because the connections between seemingly different ideas become more apparent (Knight 2004). We need to give students an authentic experience doing science before an abstraction of the scientific process will be meaningful. Starting with a hands-on experience or a discrepant event instead of the theory and explanation is a recommended approach for science instruction, because this gives students an experience to which to connect the underlying principles, and enables them to learn the explanations more deeply for having developed them themselves (Eisenkraft 2003). But even instructors who subscribe to this pedagogy for purposes of teaching students how springs work tend to start their courses with an abstraction about how science works, not noticing the disconnect.

In summary, I'm advocating moving most of the instruction about "how science works" from the beginning of courses to the middle or end, once students have done some actual science that is selfdirected and open-ended enough to be more than a set of measurements. It is then that the stories about the scientific process throughout history will be meaningful, and it is then that students will be able to build a nuanced conception of how science operates, recognizing the often-neglected importance of documenting "mistakes" and changing the project goals. Such instruction might ultimately constitute a better preparation for taking on actual science projects, such as those in the Astronomy Research Seminar.

\section{Acknowledgments}

I am unbelievably lucky to be able to work with the students of Stanford Online High School, who are my inspiration for this and everything that has come of it. In addition, I have the smartest, most supportive, and all-around incredible colleagues and mentors on the planet Earth, among whom are Gary Oas, Rachel Freed, Michael Fitzgerald, Russ Genet, and Richard Harshaw. Thanks to the Skynet Robotic Telescope Network and the Las Cumbres Observatory for generous use of their telescopes in Astronomy Research Seminar projects.

\section{References}

Crigler, C., Millar, B., and Hensley, H. (2019). WDS 09144+ 5241: CCD Observations and Orbital Solutions. JDSO, 15(1):108-118.

Eisenkraft, A. (2003). Expanding the 5E model. The Science Teacher, 70(6):56-59.

Gray, P. (2016). Inverse relationship between GPA and innovative orientation. Psychology Today.

Knight, R. D. (2004). Five easy lessons: Strategies for successful physics teaching.

McDermott, L. C., Shaffer, P. S., and Constantinou, C. P. (2000). Preparing teachers to teach physics and physical science by inquiry. Physics Education, 35(6):411. 